Introduction: HOXB8 is a protein that was found to promote cancer proliferation and invasion. ILK is a protein kinase which has a role in carcinogenesis. FAT4 is a tumor homologue that has a role in EMT and autophagy regulation.

Aim of the study: To identify expression of Human HOXB8, Integrin-linked kinase (ILK1) and FAT homolog 4 (FAT4) in colorectal cancer (CRC) correlating their expression with pathological, prognostic and clinical parameters of CRC.

Material and methods: We assessed the expression of HOXB8, ILK and FAT4 in fifty CRC patients and ten samples from nearby non-neoplastic colonic mucosa using immunohistochemistry. Results: The expression of HOXB8 and ILK in CRC was positively associated with high tumor grade, advanced tumor stage, lymph node involvement $(p<0.001)$, occurrence of distant metastases $(p=0.003$ and 0.024 respectively), higher incidence of tumor recurrence ( $p=0.03, p<0.001$ respectively), worse survival rates ( $p=0.038$ and 0.003 respectively). The expression of FAT4 in CRC was correlated with lower grade, early stage of the tumor, absence of lymph node involvement $(p<0.001)$ and lack of distant metastases $(p=0.011)$. High FAT4 expression was associated with absence of tumor recurrence $(p<0.001)$ and favorable survival rates $(p<0.001$ and 0.003$)$. Conclusions: High immunohistochemical expression of HOXB8 and ILK in addition to low immunohistochemical expression of FAT4 was associated with unfavorable prognostic and pathological parameters of CRC.

Key words: HOXB8, ILK and FAT4, colorectal cancer, recurrence, survival.

Contemp Oncol (Pozn) 2020; 24 (3): 183-192 DOI: https://doi.org/10.5114/wo.2020.100281

\section{Prognostic and clinic-pathological significances of HOXB8, ILK and FAT4 expression in colorectal cancer}

\author{
Abdulwahab A. Abuderman'1, Ola A. Harb², Loay M. Gertallah ${ }^{3}$ \\ ${ }^{1}$ Department of Basic Medical Sciences, College of Medicine, Prince Sattam Bin \\ Abdulaziz University, Al-Kharj, Saudi Arabia \\ ${ }^{2}$ Department of Pathology, Faculty of Medicine, Zagazig University, Zagazig, Egypt \\ ${ }^{3}$ Department of General Surgery, Faculty of Medicine, Zagazig University, Zagazig, Egypt
}

\section{Introduction}

Colorectal cancer (CRC) has been ranked as the $4^{\text {th }}$ cause of cancer related mortalities in the United States [1]. Nearly about 20\% of CRC patients were diagnosed late at an advanced stage. Although there is improvement in CRC treatment strategies most CRC patients experienced disease progression and recurrence which results in low survival rates and a poor clinical outcome [2]. Therefore identifying efficient novel biomarkers in addition to discovering the mechanisms of CRC early diagnosis and targeted therapy is needed to improve patients' prognosis [3]. There are many mechanisms which are involved in CRC progression and chemo-resistance and the recently incriminated mechanisms are the cancer stem cells (CSCs) and epithelial-mesenchymal transition (EMT) $[4,5]$. EMT is the main mechanisms used by cancer cells for invasion and metastases [6]. Moreover generation of cells with properties of CSC is responsible for CRC invasion, progression, metastasis and resistance to chemotherapy. Thus, targeting CSCS and EMT signaling could be able to provide a novel pathway of treatment of CRC [7]. The gene family homeobox (Hox) encodes proteins which are transcription factors and are found in nearly all eukaryotic cells [8]. There are about 39 identified Human HOX genes which are associated with development, progression and metastasis of many malignant tumors [9]. The HOX family member HOXB8 is located on 17 chromosomes. HOXB8 was found to promote cancer proliferation, invasion and metastasis through activation of EMT [2]. Integrin-linked kinase (ILK) is considered a serine/threonine protein kinase which was discovered in the inter-cellular adhesions and was found to play an essential role in interactions between cell and matrix in carcinogenesis, thus it might represent an important point of research regarding discovering novel anti-cancer targeted therapies [10]. The Fat family gene members were primarily detected in Drosophila as members of cadherin family with properties of cancer suppression [11]. FAT homolog 4 (FAT4), was first identified as a tumor suppressor homologue through EMT and autophagy regulation in many cancer cells [12].

In this study we aimed to identify the expression of the novel EMT and CSC markers; HOXB8, ILK and FAT4 in CRC and adjacent non-neoplastic tissues correlating their expression with pathological, prognostic and clinical parameters of CRC and association with progression and chemotherapy resistance.

\section{Material and methods}

To perform the current prospective study, we included samples from fifty CRC patients and ten samples from nearby non-neoplastic colonic mucosa. CRC patients were admitted and operated in General Surgery Depart- 
ment, Faculty of Medicine Zagazig University hospitals and other private hospitals where left hemi-colectomy, right hemi-colectomy or total colectomy were performed according to site of the tumor and surgically excised specimens were sent to Pathology Department Faculty of Medicine, Zagazig University where they were processed, diagnosed, subtyped, graded and staged [13].

Inclusion criteria: paraffin blocks containing sufficient samples from all CRC patients diagnosed with adenocarcinoma with different grades and stages which were suitable for immunohistochemical staining and evaluations were included.

Exclusion criteria: we excluded paraffin blocks containing insufficient material for stating, extremely necrotic samples or contain other types of cancer colon.

We acquired clinical data as age and sex of the patients before including them in the study. We obtained follow-up data of included patients during period of follow-up in the period from December 2016 to December 2019.

Ethical approval was obtained from the local ethical committee (institutional review board) of Faculty of Medicine, Zagazig University for performance of the present study.

\section{Immunohistochemistry}

Immunohistochemistry (IHC) was performed as formerly mentioned [14]. We used primary rabbit poly clonal antibodies; anti-HOXB8 antibody (Cat No. GTX51728; dilution 1 : 100), anti-FAT4 antibody (Cat No. ab130076; dilution $1: 100$ ) and anti-ILK (Cat No. ab233242 dilution 1 : 100) [2, 10, 12].

The used positive controls were basal cell carcinoma, breast carcinoma and non-neoplastic gastric mucosa tissues were done for all cases.

\section{Evaluation of HOXB8, ILK and FAT4 immunohistochemical expression}

We obtained samples from margins of the tumor or most viable areas avoiding areas of necrosis which mostly located in the center of the tumor.

HOXB8 was found and evaluated in the nuclei of tumor cells while ILK and FAT4 were found and evaluated in the cytoplasm of tumor cells. The intensity of markers expression in stained sample was scored as followed: 0, 1, 2 , and 3 points which referred to; no stain, minimal stain, moderate stain and strong stain respectively. The extent of markers expression was determined as previously described [12]; 0 (0\%), 1 (5-25\%), 2 (26-50\%), 3 (51-75\%), and 4 (76-100\%) to reach the final score of markers expression we have multiply intensity and extent score giving scores from $0-12$ taking the 4 as cut point above which is high expression and below which is high expression.

\section{Statistical analysis}

All data of patients included in the present study were collected, summarized, and statistically analyzed via using Statistical Package for Social Science (SPSS) program version 24 . We have calculated differences between qualitative variables through using $\chi^{2}$ and Fisher exact tests, correlated them using; Spearman's $\rho$ rank correlation test. The performed statistical correlations were two sided and $p$-value $\leq 0.05$ was considered significant, $p<0.001$ as considered highly significant. We analyzed correlations between HOXB8, ILK and FAT4 and collected data. Kaplan and Meier survival curves was used for estimating overall (OS) and progression free survival (PFS) rates and were stratified according to studied markers.

\section{Results}

We have included sixty tissue specimens in our study; 50 of them were retrieved from CRC patients of different histopathological subtypes, different grades and stages and 10 specimens were retrieved from adjacent non-neoplastic colonic mucosa (Table 1).

Demographic characteristics of included CRC patients were detailed in Table 2. The 50 CRC cases included $28(63.3 \%)$ males and $22(36.7 \%)$ females.

HOXB8 was expressed in CRC more than adjacent non-neoplastic colonic mucosa $(p=0.046)$. It The expression of HOXB8 in CRC was significantly associated with large tumor size, high tumor grade, advanced tumor stage, presence of lymph node $(p<0.001)$, and distant metastases $(p=0.003)$. No significant correlation was found between HOXB8 expression with initial site of the tumor, age or sex of our patients (Fig. 1).

ILK was expressed in CRC more than adjacent non-neoplastic colonic mucosa (0.009). The expression of ILK in CRC was significantly positively correlated with large tu-

Table 1. HOXB8, ILK and FAT4 expression in all studied samples

\begin{tabular}{|c|c|c|c|c|}
\hline Variables & $\begin{array}{c}\text { Adenocarcinoma }(n=50) \\
n(\%)\end{array}$ & $\begin{array}{c}\text { Normal mucosa }(n=10) \\
n(\%)\end{array}$ & $\begin{array}{c}\text { Total }(n=60) \\
n(\%)\end{array}$ & $p$-value \\
\hline \multicolumn{5}{|l|}{ HOXB8 } \\
\hline Low & $26(52.0)$ & $9(90.0)$ & $35(58.3)$ & \multirow[t]{2}{*}{0.046} \\
\hline High & $24(48.0)$ & 1 (10.0) & $25(41.7)$ & \\
\hline \multicolumn{4}{|l|}{ ILK } & \multirow{3}{*}{0.009} \\
\hline Low & $26(52.0)$ & $8(80.0)$ & $34(56.7)$ & \\
\hline High & $24(48.0)$ & $2(20.0)$ & $26(43.3)$ & \\
\hline \multicolumn{4}{|l|}{ FAT4 } & \multirow{3}{*}{$<0.001$} \\
\hline Low & $33(66.0)$ & $0(0.0)$ & $33(55.0)$ & \\
\hline High & $17(34.0)$ & $10(100.0)$ & $27(45.0)$ & \\
\hline
\end{tabular}




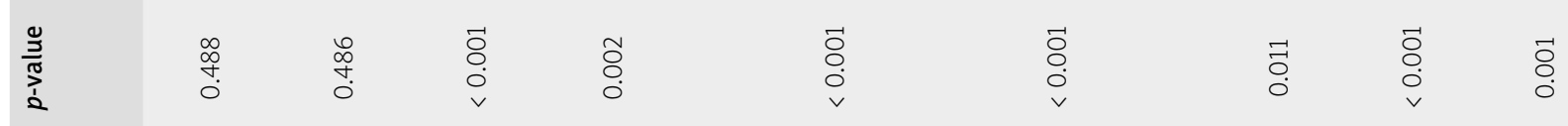
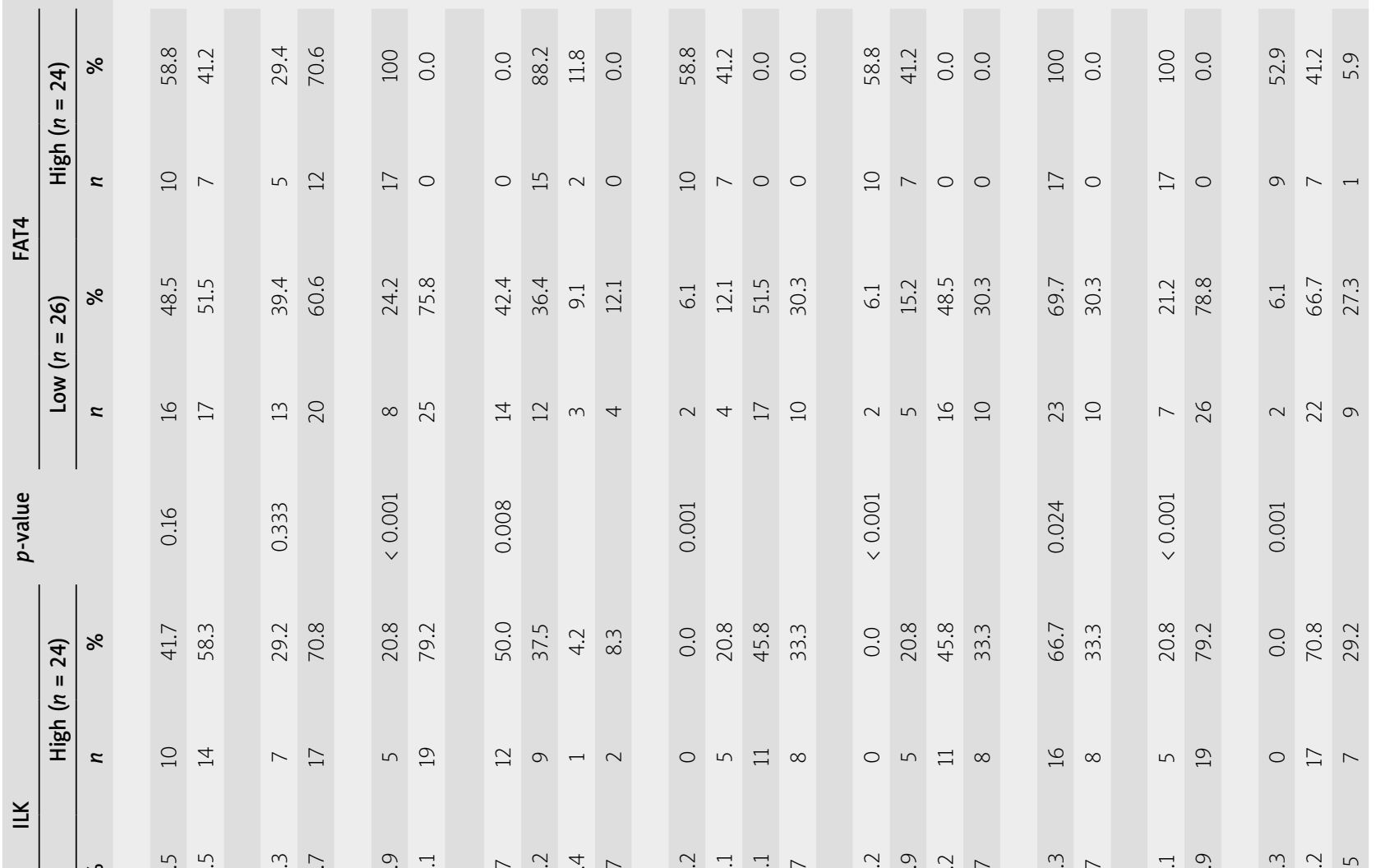

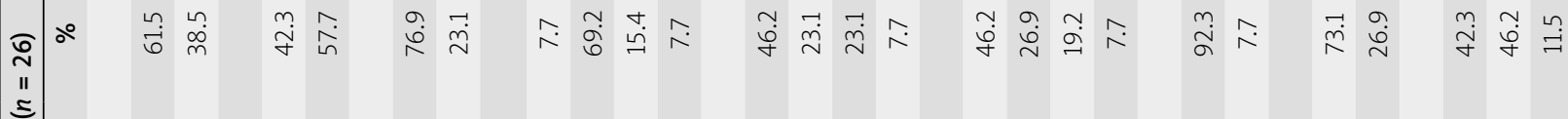

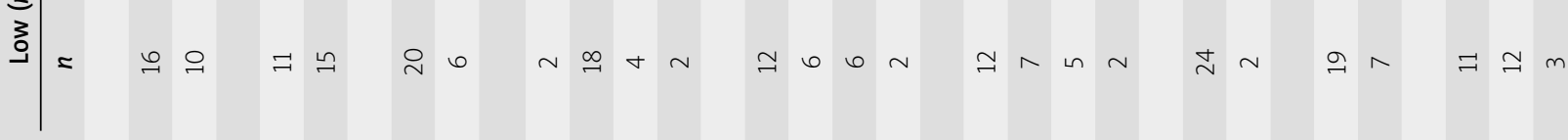

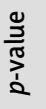

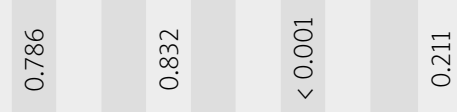

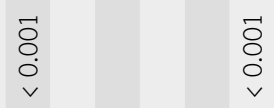
$\begin{array}{llll}n & \overrightarrow{0} & \overrightarrow{0} \\ 0 & 0 & 0 \\ 0 & 0 & 0\end{array}$

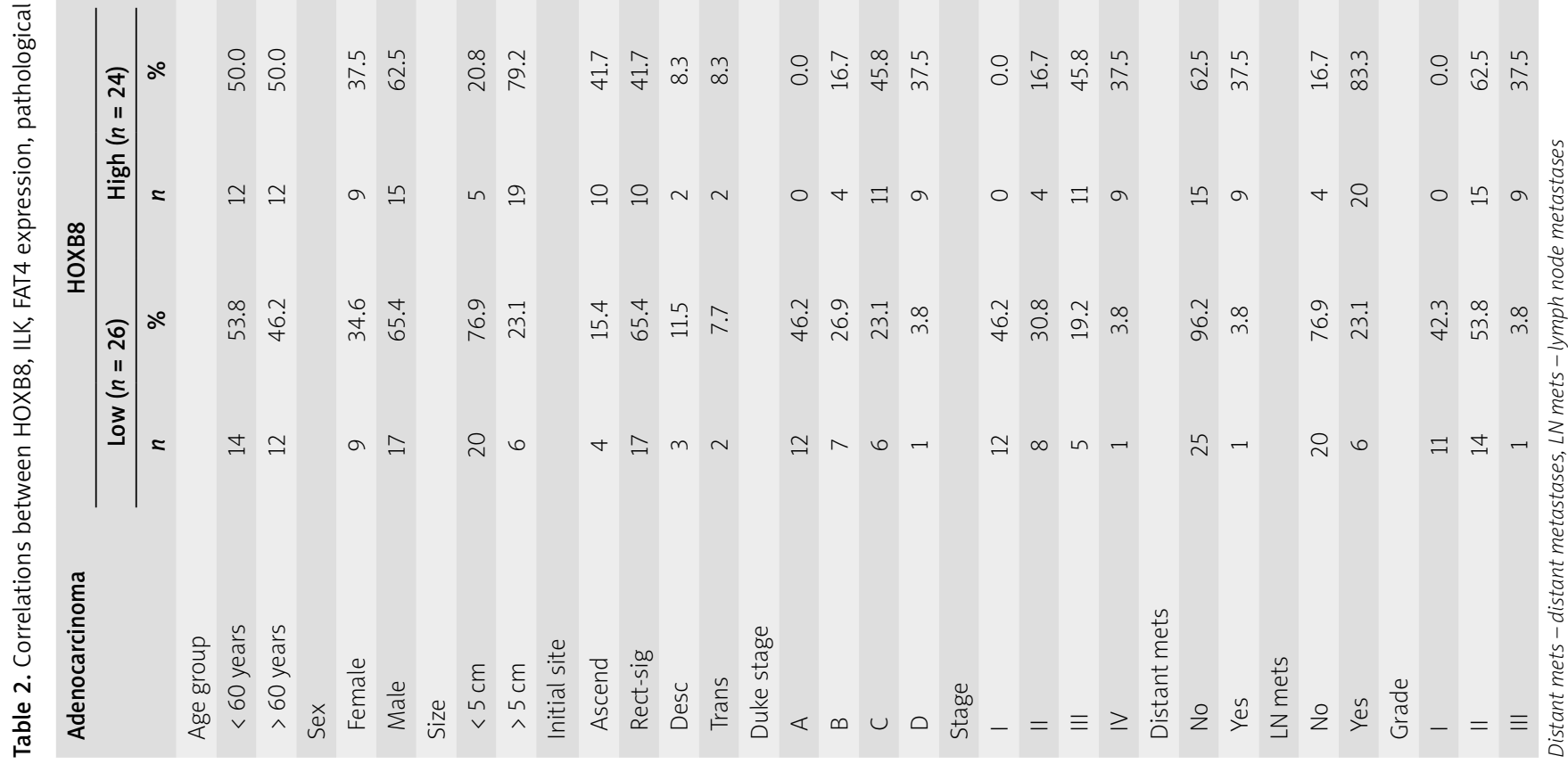



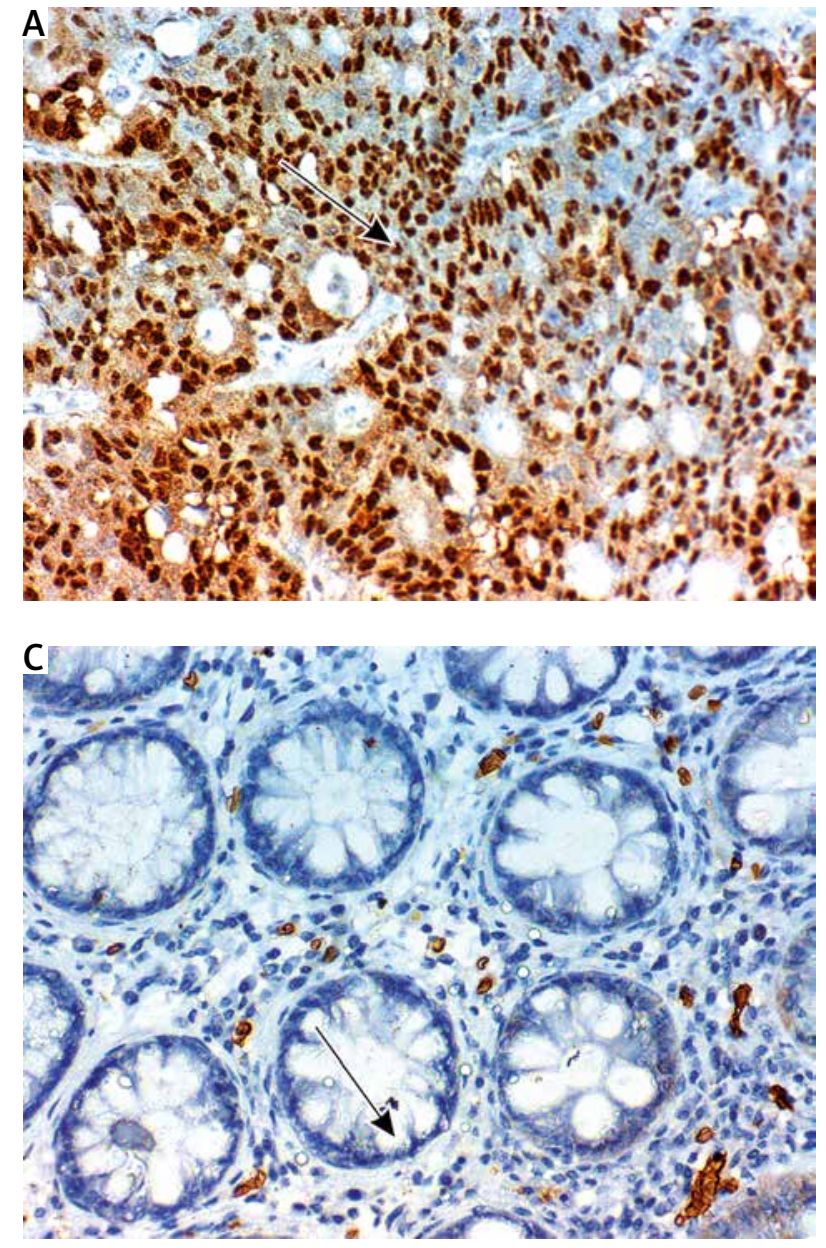

mor size, high tumor grade, advanced tumor stage, presence of lymph node $(p<0.001)$ and distant metastases $(p=0.024)$. No significant correlation was found between ILK expression, histopathological subtype of the lesion age or sex of our patients (Fig. 2).

FAT4 was down regulated in CRC more than adjacent non-neoplastic colonic mucosa $(p<0.001)$. The expression of FAT4 in CRC was associated with smaller size of the tumor, lower grade, early stage of the tumor, absence of lymph node $(p<0.001)$ and distant metastases $(p=0.011)$. No significant correlation was found between FAT4 expression and age or sex of our patients (Fig. 3).

High HOXB8 expression was significantly associated with poor response to therapy $(p=0.026)$, higher incidence of recurrence after successful treatment $(p=0.03)$, worse 3-year DFS and OS rates ( $p=0.038$ and 0.003 respectively). High ILK expression was significantly positively correlated with; poor response to therapy ( $p=0.039$ ), high incidence of recurrence of the tumor after successful treatment $(p<0.001)$, worse 3 -year DFS and OS rates ( $p<0.001$ and 0.005 respectively). High FAT4 expression was significantly associated with absence of recurrence of the tumor after successful therapy $(p<0.001)$, good response to therapy $(p=0.022)$, favorable 3 -year DFS and OS rates ( $p<0.001$ and $p=0.003)$. HOXB8 and ILK expression are positively correlated with each other $r=0.439(p<0.001)$

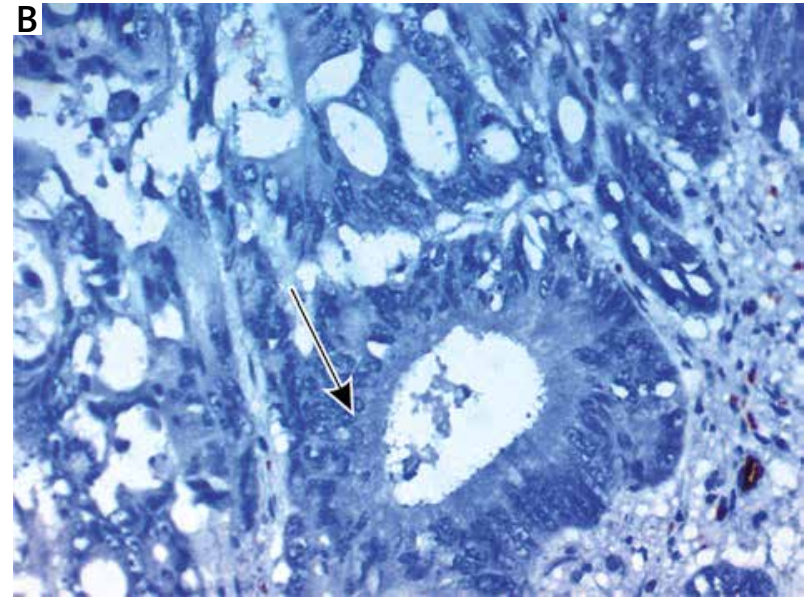

Fig. 1. Expression of HOXB8 in nuclei of cells of colorectal carcinoma (CRC). A) High expression in high grade and stage CRC $\times 400$, B) totally negative expression in CRC grade 2 , stage 1 CRC $\times 400$, C) negative expression in non-neoplastic colonic mucosa $\times 400$. The arrows point to site of positive or negative nuclear expression of the maker

and both are negatively associated with FAT4 expression $r=-0.521(p<0.001)$ (Figs. 4 and 5, Tables 3 and 4).

\section{Discussion}

In the current study we tried to clarify the role of EMT and CSCs markers in CRC progression by evaluation of expression of three markers that were incriminated in EMT induction; HOXB8, ILK and FAT4. HOX genes were recently found to play a role in cancer occurrence, development, invasion and metastasis [8, 15], particularly in CRC of different stages [16]. However, the role of HOXB8; a Hox gene family member is still inconclusive. In our study we have confirmed that up-regulation of HOXB8 is found in CRC tissues more than adjacent mucosa. HOXB8 expression promoted progression and metastases of CRCs, which is similar to results of Wang et al. [2], who found the same results in CRC and results of Ding et al. [9] in gastric cancer. Similarly Wang et al. [2] found that HOXB8 overexpression promotes CRC proliferation and invasion, while its knocking down leads to occurrence of opposite effects. EMT is a major mechanism which is incriminated in cancer cells migration and invasion [17]. Ding et al. have showed that HOXB8 increased gastric cancer cells spread by EMT induction [9]. This observation indicated that HOXB8 promoted CRC cells invasion, lymphatic and hematogenous metastasis that subsequently leads to poor patients' prognosis. 

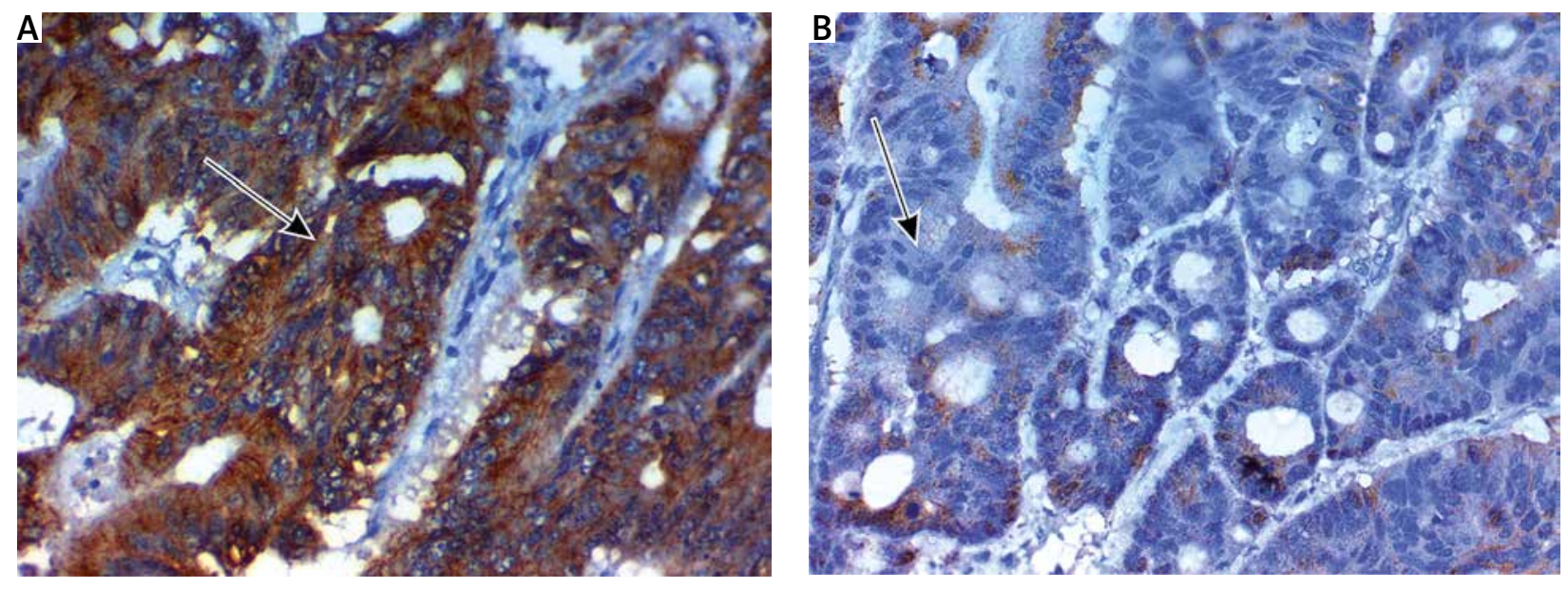

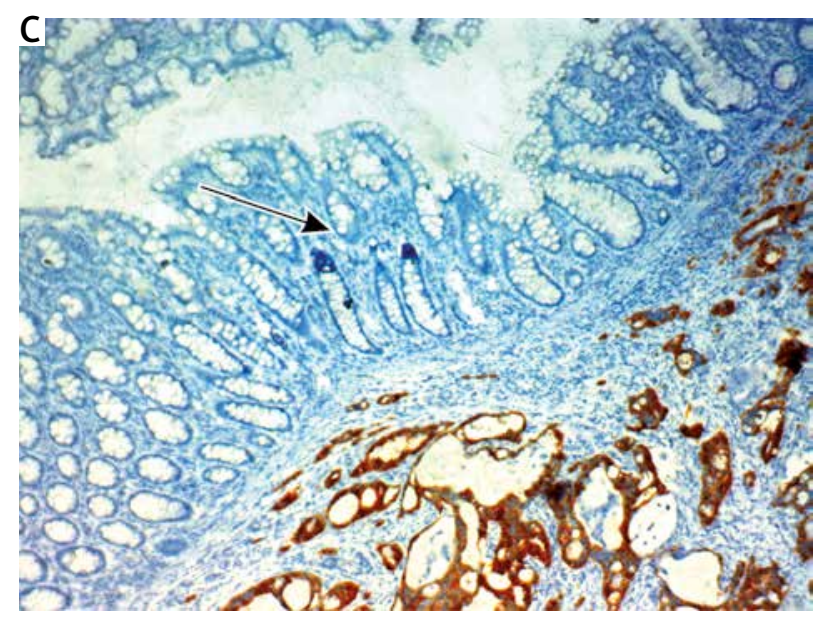

A possible mechanism of action was showed by Wang et al. [2], that increased HOXB8 expression significantly activate the phosphorylated-STAT3 and STAT3 which cooperated with Twist to induce malignant invasion and metastasis through EMT induction. HOXB8 could lead to induction of EMT via STAT3 pathway activation in CRC cells denoting the essential connection between EMT and HOXB8 [18]. We showed that HOXB8 overexpression is related to chemo-resistance which is similar to results of $\mathrm{Lu}$ et al. who established that HOXB8 is accurate in predicting FOLFOX4 chemo-sensitivity in CRC patients with liver metastases [19]. Advanced CRC patients with increased HOXB8 expression have stable or progressive disease, while patients with low expression have partial response [19]. Ding et al. [9] showed that HOXB8 played a major role in EMT induction in gastric cancer and distant metastasis, additionally, the expression of HOXB8 in gastric cancer cells was more than non-neoplastic mucosa [9]. A previous study has shown that overexpression of HOX8 increased tumor cell proliferation [20]. Ding et al. [9] pointed to that HOXB8 not only induce EMT, but its inhibition leads to MET. Due to few studies regarding roles of HOXB8 in CRC we assessed the expression of other biomarkers to prove its roles. We have assessed the expression of ILK as previous studies showed that ILK is implicated in CRC carcinogenesis and represented an important Wnt/ $\beta$-catenin pathway regulation [10]. ILK's was previously found to be
Fig. 2. Expression of ILK in cytoplasm of cells of colorectal carcinoma (CRC). A) High expression in high grade and stage $C R C \times 400$, B) low expression in low grade and stage $C R C \times 400, C)$ negative expression in non-neoplastic colonic mucosa $\times 400$. NB in Fig. $2 \mathrm{C}$ we found some cells with high expression in the stroma but that was nonspecific staining and we evaluated only markers expression in epithelial cells. The arrows point to site of positive or negative cytoplasmic expression of the maker

involved in CRC carcinogenesis through induction of EMT and causing chemo-resistance in many cancers, so we hypothesized that ILK might have a role in CRC progression and chemo- resistance [20,21].

In the present study we stated that overexpression of ILK in CRC was related to poor prognosis, dismal outcome, cancer progression and chemo-resistance which was similar to Tsoumas et al. [10]. We showed that ILK overexpression in CRC is related to high grade, advanced stage and it is considered a predictor of lymph node metastasis proving its role in CRC progression [10, 22]. Moreover, we showed that ILK expression in our samples is positively correlated with HOXB8 expression that is an EMT biomarker, similarly Tsoumas et al. [10] showed that ILK is associated with EMT and CSCs biomarkers in CRC cells and that expression of ILK was related to altered expression of $\beta$-catenin and E-cadherin. Collectively these results suggested that high expression of ILK induced CSC and EMT phenotype in CRC cells and related to tumor progression as was previously stated [23]. Moreover, Tsoumas et al. found that inhibition of ILK in CRC cells inhibit the main EMT regulator Snail [10]. ILK was found to stimulate stem cell characteristics in cancer cells [24]. We showed that ILK overexpression is related to poor response to chemotherapy. In agreement with our results, ILK has been incriminated in chemo- resistance in other malignancies as cancer lung and glioma [21, 22]. Our data and results of previous studies pointed to that 

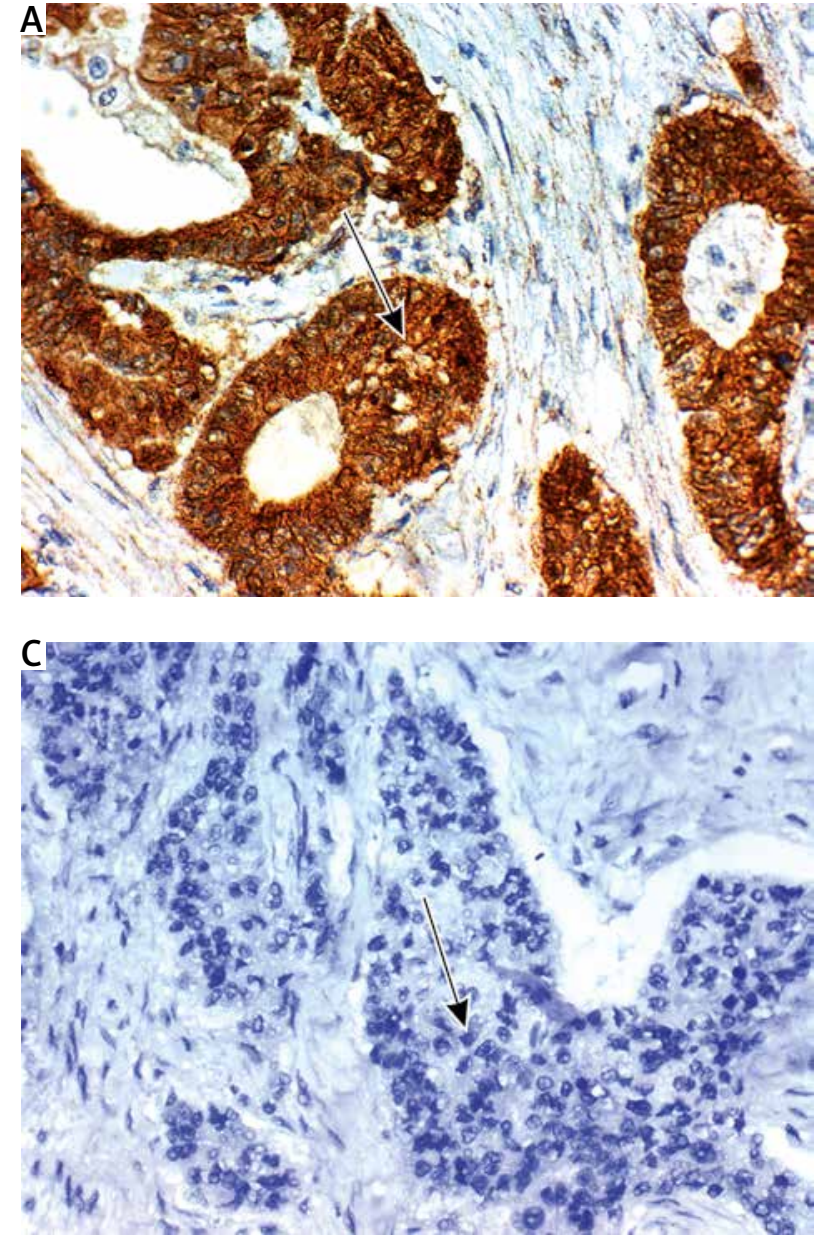

ILK targeting ILK could have a synergistic effect with currently used chemotherapeutic agents in many cancers and might be able to reduce chemo-resistance in gastric cancer $[25,26]$.

Autophagy is a homeostatic mechanism that occurs in response to cellular stress and it involved engulfment and recycling of unneeded cellular organelles and cytoplasmic components via lysosomal degradation pathways to maintain cellular metabolism [27]. Effect of autophagy in malignant cells in cancer progression remains controversial [28]. Autophagy inhibition could exert many effects on EMT, and FAT4 roles in autophagy in CRCs require clarification.

We assessed the expression of FAT4 in CRC and adjacent non-neoplastic tissues due its role in autophagy and correlating its expression with studied EMT and CSCS biomarkers; HOXB8 and ILK. We found that FAT4 was down-regulated in CRC cells than adjacent non-neoplastic colonic mucosa and loss of its expression was related to CRC progression which is similar to Wei et al. [12] in CRC and Jiang et al. [11] in gastric cancer. In the Jiang et al. [11], study, loss of FAT4 expression was related to poor prognosis, larger tumor size, deeper invasion, and presence of vascular and lymphatic invasion, lymph node and distant metastases of gastric cancer and they stated that levels of FAT4 mRNA were lower in cancer tissues than adjacent

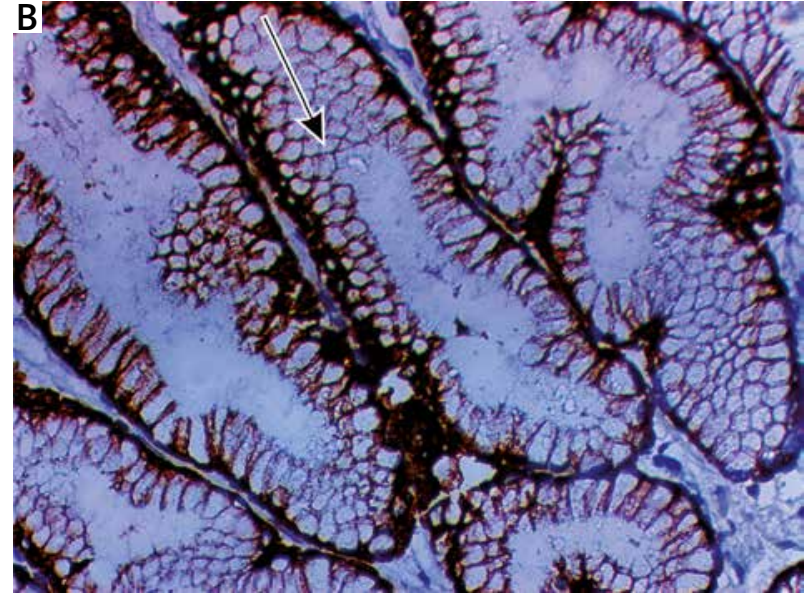

Fig. 3. Expression of FAT4 in cytoplasm of cells of colorectal carcinoma (CRC). A) High expression in low grade and stage CRC $\times 400$, B) low expression in high grade and stage CRC $\times 400$, C) negative expression in non-neoplastic colonic mucosa $\times 400$. The arrows point to site of positive or negative membranous expression of the maker

non neoplastic gastric tissues which was near our results in CRC.

Our data is in line with former reports studies which showed the tumor suppressive role of FAT4 in many malignancies $[29,30]$.

Our results were explained by those Fat gene family members particularly FAT4 were considered cadherin superfamily members that controls cellular adhesion, proliferation and Hippo signaling pathway [29, 31]. Disturbances in Hippo pathway are related to development and progression of cancer [32]. Collectively we found that low expression of FAT4 in CRC is associated with its progression which resulted in poor prognosis and that FAT4 is a novel prognostic biomarker and therapeutic target for patients with CRC [33].

FAT4 was previously found to increase expression of $\mathrm{E}$-cadherin and decrease $\mathrm{N}$-cadherin and Twist1 expression which leads to EMT inhibition $[12,33]$. These results were near our results that FAT4 expression was inversely associated with HOXB8 and ILK expression which were EMT and CSCs biomarkers [12, 33]. Thus, our study highlighted the inverse association between autophagy, EMT and CSCs in CRC tissues. Autophagy is pathway which is responsible for engulfment, digestion in addition to recycling intracellular organelles to produce energy through lysosomal degradation [34], additionally it could reduce cell damage and maintain cellular viability under stressful 

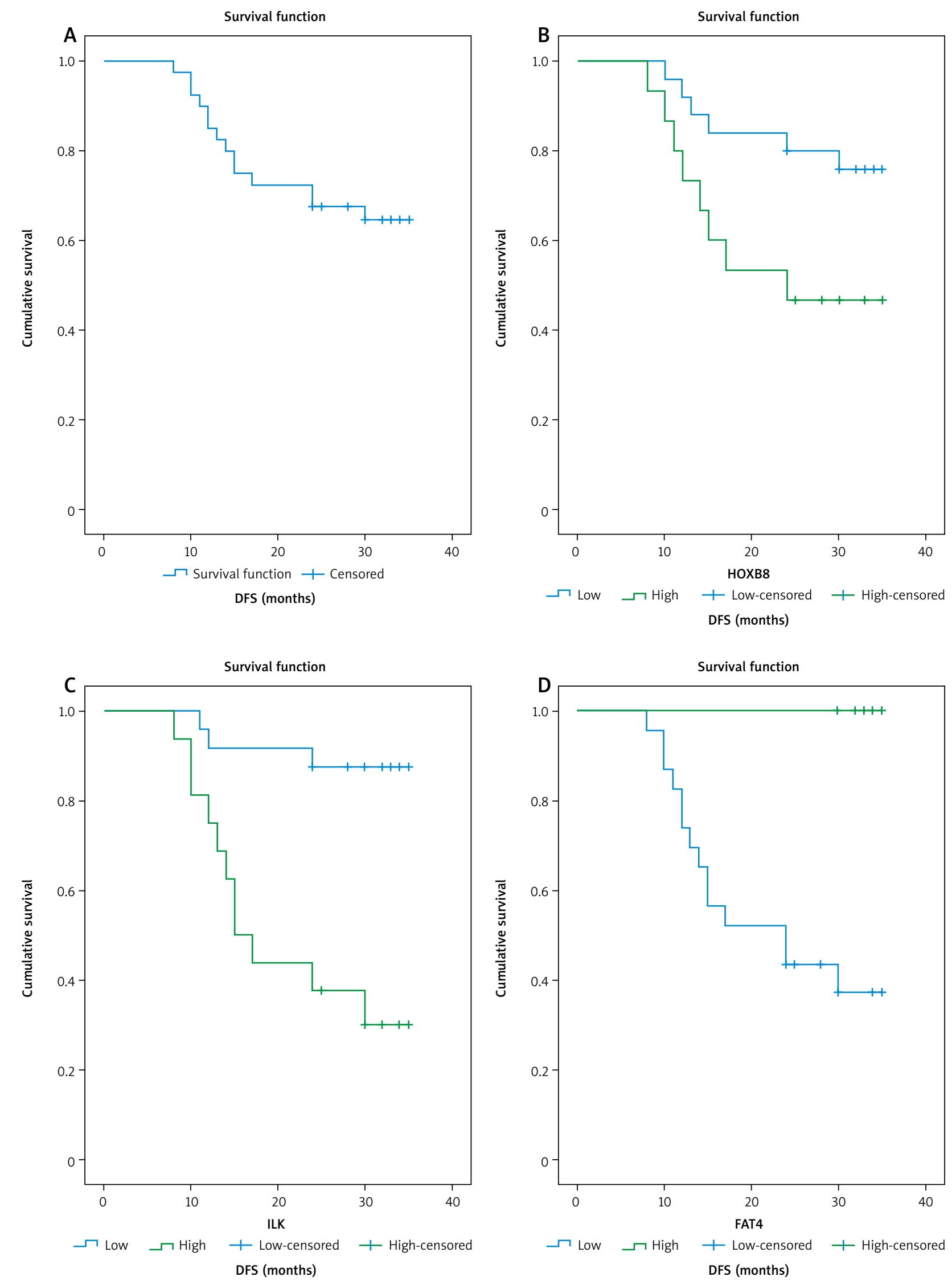

DFS (months)

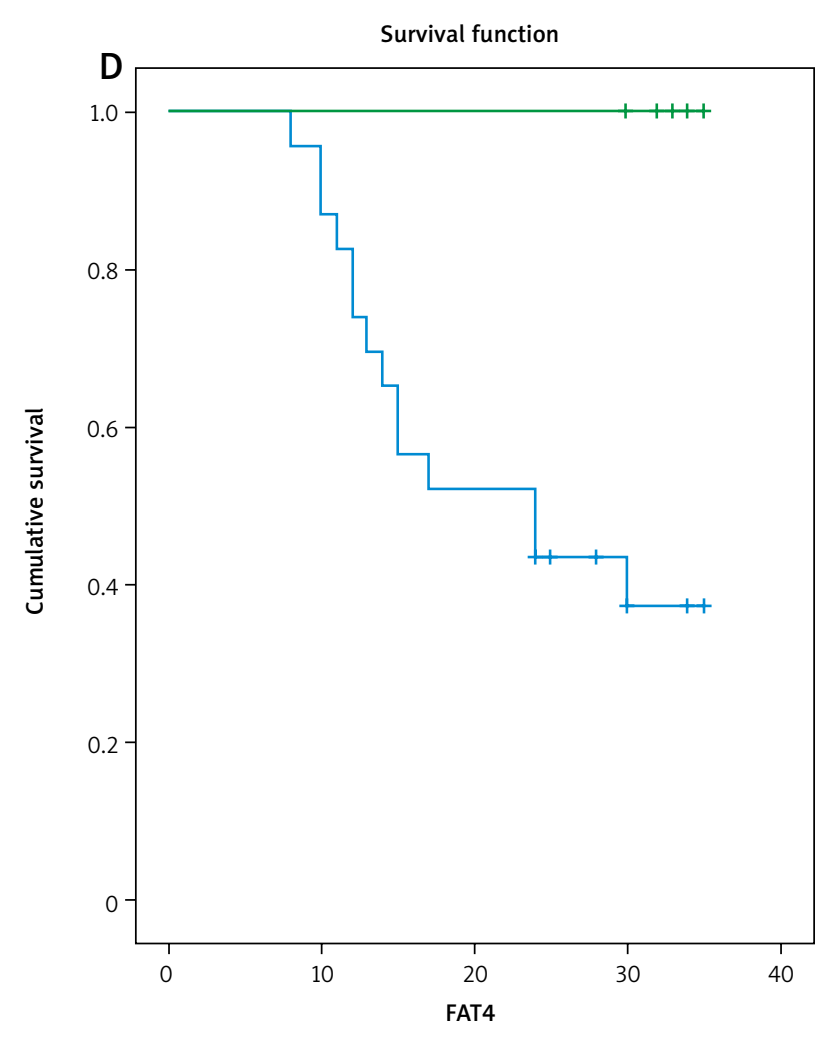

Fig. 4. Kaplan Meir survival curves of disease-free survival (DFS) of the studied colorectal carcinoma (CRC) patients. A) DFS rate of all the studied CRC cases, B) DFS rate stratified according to HOXB8 expression, C) DFS rate stratified according to ILK expression, D) DFS rate stratified according to FAT4 expression 

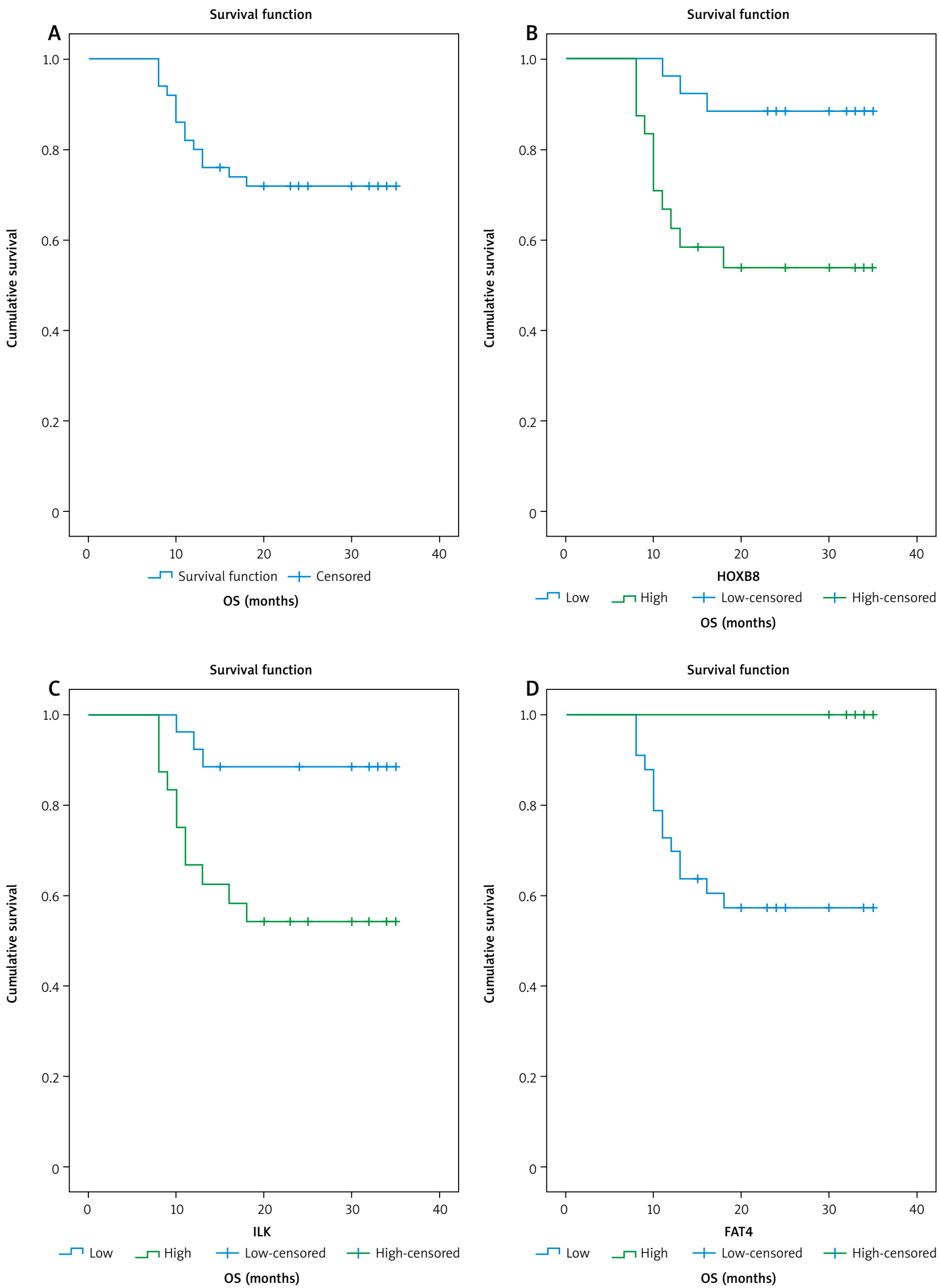

Fig. 5. Kaplan Meir survival curves of overall survival rate (OS) of the of the studied colorectal carcinoma (CRC) patients. A) OS rate of all the studied CRC cases, B) OS rate stratified according to HOXB8 expression, C) OS rate stratified according to ILK expression, D) OS rate stratified according to FAT4 expression 


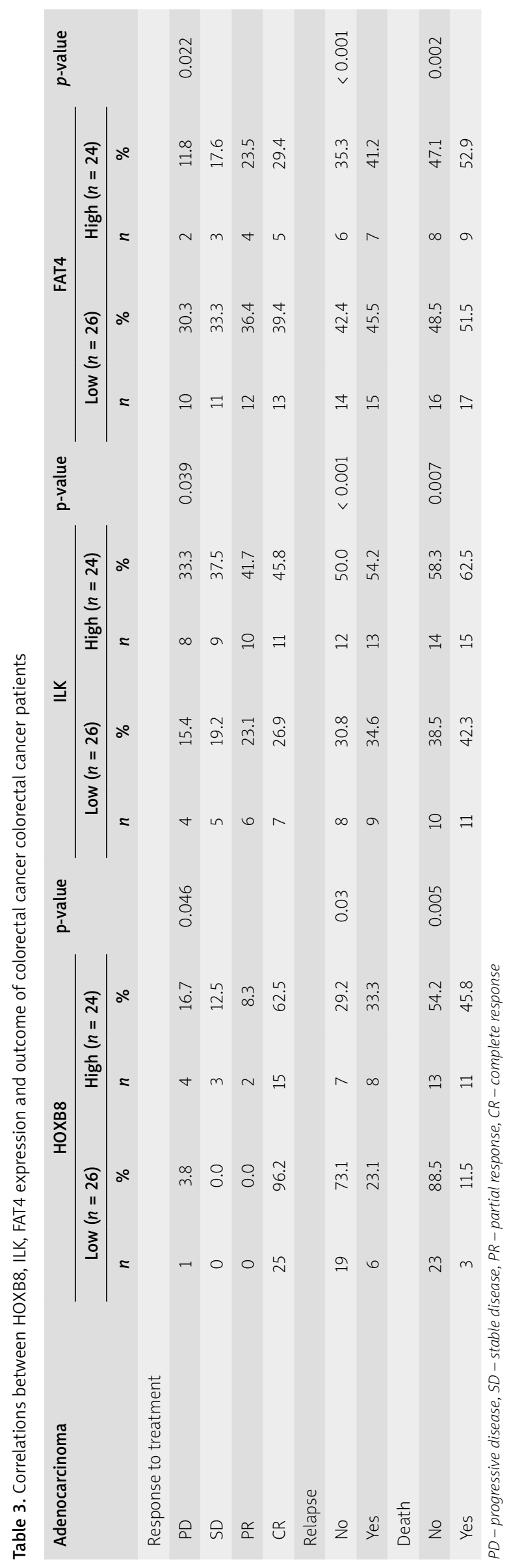

Table 4. Correlations between HOXB8, ILK, FAT4 expression disease-free survival and overall survival in colorectal cancer patients

\begin{tabular}{lcccc}
$\begin{array}{l}\text { Variables } \\
\text { HOXB8 }\end{array}$ & 3-year DFS (\%) & $p$-value & 3-year OS (\%) & $p$-value \\
\hline Low & 75.8 & 0.038 & 88.5 & 0.003 \\
High & 46.7 & & 53.8 & \\
ILK & & & & \\
Low & 87.5 & $<0.001$ & 88.5 & 0.005 \\
High & 30 & & 54.2 & \\
FAT4 & & & & \\
Low & 37.3 & $<0.001$ & 57.3 & 0.003 \\
High & 100 & & 100 & \\
\hline DFS - disease-free survival rate, OS- overall survival rate &
\end{tabular}

conditions. The normal cells in comparison to cancer cells might face many stresses and were more dependent on autophagy so they express more autophagy biomarkers [35]. FAT4 promoted autophagy in CRC cells so reduced its progression. In Wei et al. [12] study, they observed that FAT4 promoted autophagy in cancer cells. Egan et al. [33] and Byers et al. [36] showed that FAT4 has a role in inhibition of EMT. Moreover inhibition of FAT4 could be able to activate Yes-associated protein (YAP) and WNT signaling pathways so it is responsible for cancer progression [11].

Up to our knowledge we are the first study that tried to identify roles of the expression of HOXB8, ILK and FAT4 in CRC. However, there are several limitations. The study included few samples of studied CRC and normal mucosa samples, with evaluation of the markers expression using immunohistochemistry only. Selection bias was existed during inclusion of cases due to little number of cases and design of the study to perform assessment of the markers semi-quantitatively by immunohistochemistry and not by other methods like genetic evaluation.

\section{Conclusions}

We found that up-regulation of HOXB8 and ILK in addition to down-regulation of FAT4 was found in CRC tissues more than adjacent non-neoplastic colonic mucosa and was associated with unfavorable prognostic and pathological parameters pointing to the possibility of using these markers as novel prognostic biomarkers and therapeutic targets for CRC to improve its prognosis and management strategies.

The authors declare no conflict of interest.

\section{References}

1. Siegel RL, Miller KD, Fedewa SA, Ahnen DJ, Meester RG, Barzi A, Jemal A. Colorectal cancer statistics, 2017. CA Cancer J Clin 2017; 67: 177-193.

2. Wang T, Lin F, Sun X, Jiang L, Mao R, Zhou SH et al. HOXB8 enhances the proliferation and metastasis of colorectal cancer cells by promoting EMT via STAT3 activation Cancer Cell Int 2019; 19: 3.

3. Van Cutsem E, Cervantes A, Adam R, et al. ESMO consensus colorectal cancer. Ann Oncol 2016; 27: 1386-1422. 
4. Wenzel ES, Singh ATK. Cell-cycle checkpoints and aneuploidy on the path to cancer. In Vivo 2018; 32: 1-5.

5. Sato R, Semba T, Saya H, Arima Y. Concise review: stem cells and epithelial-mesenchymal transition in cancer: biological implica tions and therapeutic targets. Stem Cells 2016; 34: 1997-2007.

6. Thiery JP. Epithelial-mesenchymal transitions in tumor progression. Nat Rev Cancer 2002; 2: 442-454.

7. Jung HJ. Chemical proteomic approaches targeting cancer stem cells: a review of current literature. Cancer Genom Proteom 2017; 14: 315-332.

8. Haria D, Naora H. Homeobox gene deregulation: impact on the hallmarks of cancer. Cancer Hallm 2013; 1: 67-76.

9. Ding WJ, Zhou M, Chen MM, Qu CY. HOXB8 promotes tumor me tastasis and the epithelial-mesenchymal transition via ZEB2 targets in gastric cancer. J Cancer Res Clin Oncol 2017; 143: 385-397.

10. Tsouma D, Nikou S, Giannopoulou E, et al. ILK Expression in Colorectal Cancer Is Associated with EMT, Cancer Stem Cell Markers and Chemo-resistance. Cancer Genom Proteom 2018; 15: 127-141.

11. Jiang X, Liu Z, Xia Z, et al. Low FAT4 expression is associated with a poor prognosis in gastric cancer patients Oncotarget 2018; 9: 5137-5154.

12. Wei R, Xiao Y, Song Y, Yuan H, Luo Y, Xu W. FAT4 regulates the EMT and autophagy in colorectal cancer cells in part via the PI3KAKT signaling axis. Cancer Genom Proteom 2018; 15: 127-141.

13. Amin MB, Edge S, Greene F, et al. AJCC Cancer Staging Manual. $8^{\text {th }}$ ed. Springer, New York 2017.

14. Hsu SM, Raine L, Fanger H. Use of avidin-biotin-peroxidase complex $(A B C)$ in immunoperoxidase techniques: a comparison be tween $A B C$ and unlabeled antibody (PAP) procedures. J Histochem Cytochem 1981; 29: 577-580.

15. Hur H, Lee JY, Yun HJ, Park BW, Kim MH. Analysis of HOX gene expression patterns in human breast cancer. Mol Biotechnol 2014 56: 64-71.

16. Liao WT, Jiang D, Yuan J, et al. HOXB7 as a prognostic factor and mediator of colorectal cancer progression. Clin Cancer Res 2011; 17: 3569-3578

17. Antognelli C, Cecchetti R, Riuzzi F, Peirce MJ, Talesa VN. Glyoxalase 1 sustains the metastatic phenotype of prostate cancer cells via EMT control. J Cell Mol Med 2018; 22: 2865-2883.

18. Fu Q, Jiang Y, Zhang D, Liu X, Guo J, Zhao J. Valosin-containing protein (VCP) promotes the growth, invasion, and metastasis of colorectal cancer through activation of STAT3 signaling. Mol Cell Biochem 2016; 418: 189-198.

19. Lu X, Pan J, Li S, Shen S, Chi P, Lin H, Huang Y, Xu Z, Huang S. Establishment of a predictive genetic model for estimating chemotherapy sensitivity of colorectal cancer with synchronous liver metastasis. Cancer Biother Radiopharm 2013; 28: 552-558.

20. Liu YJ, Zhu Y, Yuan HX, Zhang JP, Guo JM, Lin ZM. Overexpression of HOXC11 homeobox gene in clear cell renal cell carcinoma in duces cellular proliferation and is associated with poor prognosis. Tumour Biol 2015;36: 2821-2829.

21. Jia Z. Role of integrin-linked kinase in drug resistance of lung cancer. Onco Targets Ther 2015; 8: 1561-1565

22. Liang F, Wang B, Bao L, Zhao YS, Zhang SM, Zhang SO. Overexpression of ILK promotes temozolomide resistance in glioma cells. Mo Med Rep 2017; 15: 1297-1304.

23. Bravou V, Klironomos G, Papadaki E, Stefanou D, Varakis J. Integ rin-linked kinase (ILK) expression in human colon cancer. Br J Cancer 2003; 89: 2340-2341.

24. Rothe K, Babaian A, Nakamichi N, et al. The focal adhesion complex Ilk-pinch-parvin is key to niche interactions and survival of quiescent stem cells in chronic myeloid leukemia in vitro and in vivo. Blood 2016; 128: 936

25. Kalra J, Warburton C, Fang K, et al. QLT0267, a small molecule inhibitor targeting integrin-linked kinase (ILK), and docetaxel can combine to produce synergistic interactions linked to enhanced cytotoxicity, reductions in P-AKT levels, altered F-actin architecture and improved treatment outcomes in an orthotopic breast cancer model. Breast Cancer Res 2009; 11: R25.

26. Song $W$, Jiang R, Zhao CM. Role of integrin-linked kinase in multidrug resistance of human gastric carcinoma SGC7901/ DDP cells. Asian Pac J Cancer Prev 2012; 13: 5619-5625.
27. Mathew R, Karantzawadsworth V, White E. Role of autophagy in cancer. Medical Recapitulate 2010; 7: 961.

28. Karantzawadsworth V, Patel S, Kravchuk O, Chen G, Mathew R, Jin S, White E. Autophagy mitigates metabolic stress and genome damage in mammary tumorigenesis. Genes Dev 2007; 21: 16211635.

29. Qi C, Zhu YT, Hu L, Zhu YJ. Identification of Fat4 as a candidate tumor suppressor gene in breast cancers. Int J Cancer 2009; 124 : 793-798.

30. Katoh M. Function and cancer genomics of FAT family genes (review). Int J Oncol 2012; 41: 1913-1918.

31. Cai J, Feng D, Hu L, Chen H, Yang G, Cai Q, Gao C, Wei D. FAT4 functions as a tumour suppressor in gastric cancer by modulating Wnt/beta-catenin signalling. Br J Cancer 2015; 113: 1720-1729.

32. Mo JS, Park HW, Guan KL. The Hippo signaling pathway in stem cell biology and cancer. EMBO Rep 2014; 15: 642-656.

33. Egan D, Chun MH, Vamos M, et al. Small molecule inhibition of the autophagy kinase ULK1 and identification of ULK1 substrates. Mol Cell 2015; 59: 285-297.

34. Choi AMK, Ryter SW, Levine B. Autophagy in human health and disease. N Engl J Med 2013; 368: 651-662.

35. Kroemer G, Mariño G, Levine B. Autophagy and the integrated stress response. Mol Cell 2010; 40: 280-293.

36. Byers LA, Diao L, Wang J, et al. An epithelial-mesenchymal transition (EMT) gene signature predicts resistance to EGFR and PI3K inhibitors and identifies Axl as a therapeutic target for overcoming EGFR inhibitor resistance. Clin Cancer Res 2013; 19: 279-290.

\section{Address for correspondence}

\section{Ola A. Harb}

Department of Pathology

Faculty of Medicine

Zagazig University

11111 Zagazig, Egypt

e-mail: olaharb2015@gmail.com

Submitted: 8.08.2020

Accepted: 11.10 .2020 